\section{INFLUÊNCIA DA REFLEXOLOGIA PODAL NA QUALIDADE DO SONO: ESTUDO DE CASO}

\section{INFLUENCE OF FOOT REFLEXOLOGY IN SLEEP QUALITY: CASE STUDY}

\section{RESUMO}

O sono é o resultado de um mecanismo complexo, envolvendo a ação conjunta de várias áreas do sistema nervoso. $O$ ciclo sono-vigília é essencial para restaurar as funções orgânicas. Na sociedade moderna, as atividades diárias produtoras de estresse podem levar ao desenvolvimento da insônia. A insônia é definida pela dificuldade de iniciar e ou manter o sono, presença de sono não reparador, de quantidade inadequada ou de qualidade insatisfatória, durante um período não inferior a um mês. A insônia primária está associada ao alto nível de alerta fisiológico e psicológico durante a noite e condicionamento negativo para dormir. $\mathrm{O}$ tratamento inclui terapias farmacológicas e não farmacológicas, entre estas, encontra-se a reflexologia podal, uma técnica de massoterapia que, através de pressões em diferentes regiões dos pés, atinge reflexamente os órgãos correspondentes, revitalizando-os e reequilibrando o organismo. $\mathrm{O}$ objetivo deste trabalho foi verificar os efeitos da técnica de reflexologia podal na qualidade do sono, na forma de estudo de caso, em três voluntárias que receberam entre seis a oito sessões da técnica completa, por 30 minutos, semanalmente. Foi utilizado como instrumento de avaliação o Índice de Qualidade do Sono de Pittsburgh (IQSP) imediatamente antes e após a realização das sessões. Os resultados mostraram um efeito positivo na melhora da qualidade do sono em duas voluntárias, demonstrando que esta técnica de massagem pode ser um promissor recurso complementar à abordagem biomédica. Estudos devem ser realizados para um maior entendimento a respeito de seu mecanismo de ação e padronização dos resultados.

\section{PALAVRAS-CHAVE}

Reflexologia podal.

Sono.

Transtornos do sono.
CORRESPONDENTE

Fatima Aparecida Vieira Machado

Endereço: Av. Huberto Bruning, n.360, Jardim Santos Dumont, Paranavaí

- Paraná. CEP.: 87706-490.

E-mail: fatimavm@unipar.br

Recebido: 02/07/13

Aprovado: 12/03/14 


\begin{abstract}
Sleep is the result of a complex mechanism involving the join action of several areas of the nervous system. The sleep-wake cycle is essential to restore organ functions. In modern society, the daily activities producing stress can trigger insomnia. The insomnia is defined as difficulty initiating sleep and/or maintaining sleep, presence of non-restorative sleep, inadequate and poor quality for a period not less than one month. Primary insomnia is associated with a high level of alertness physiological and psychological overnight and negative conditioning to sleep. The treatment consists of pharmacological and nonpharmacological therapies, among these, is the foot reflexology, a technique of massage therapy which through pressure on different regions of the feet, reaches reflex the corresponding organs, revitalizing and rebalancing the body. The aim of this study was to determine the effects of foot reflexology technique on sleep quality in the form of case study in three volunteers who received six to eight complete sessions of technique for 30 minutes, weekly. The index the Pittsburgh Sleep Quality (PSQI) was used as an evaluation tool, immediately before and after the sessions. The results showed a positive effect in improving the quality of sleep in two volunteers, demonstrating that this technique of massage can be a promising feature complementary to conventional medical treatment. Studies should be conducted to a greater understanding about its mechanism of action and standardization of results.
\end{abstract}

KEY WORDS: Foot reflexology. Sleep. Sleep disorders.

\section{INTRODUÇÃO}

O sono é um estado fisiológico caracterizado pela suspensão reversível do estado de consciência, sendo o resultado de um mecanismo complexo, envolvendo a ação conjunta de várias áreas do sistema nervoso. O ciclo sono-vigília é fundamental para restaurar as funções orgânicas. ${ }^{1,2}$

Para McCrae et al ${ }^{3}$, Müller e Guimarães ${ }^{4}$; Palma et al ${ }^{5}$, a duração insuficiente, ou a baixa qualidade do sono compromete a capacidade física e cognitiva, levando à perda de motivação no trabalho, com consequente perda da produtividade, além de riscos com a segurança própria e a dos outros e, ocasionando desgaste das relações no ambiente familiar e social, podendo levar ao desenvolvimento de diferentes doenças.

A qualidade do sono não esta propriamente associada a horas de sono, mas à profundidade do sono, ao número de despertares noturno e, à adequada preparação do organismo para as atividades ao acordar e, ainda, à satisfação com o trabalho e, à produtividade. ${ }^{6}$

Estima-se que 25-30\% dos adultos possuam dificuldades para dormir ocasionalmente e cerca de $10 \%$ apresentam a insônia crônica. ${ }^{7}$
Para Ohayon $^{8}$, a insônia caracteriza-se pela dificuldade em iniciar e/ou manter o sono e a sensação de não ter tido um sono reparador, por um período não inferior a um mês.

Monti', Robaina et $\mathrm{a}^{10}$, afirmam que a insônia primária está associada ao alto nível de estresse fisiológico e psicológico durante a noite e, também, observa-se um condicionamento negativo para dormir. Preocupações intensas e mal estar relacionados à impossibilidade de dormir geram um círculo vicioso, que pode levar à cronificação do processo ocasionando distúrbios de memória e de concentração, falta de ânimo e fadiga, podendo levar a um quadro de depressão, além de problemas cardiovasculares e disfunções metabólicas.

As dificuldades econômicas, os problemas familiares, entre outros, fazem parte de eventos da vida considerados produtores de estresse que podem desencadear a insônia. ${ }^{9,11}$

A insônia reflete, além de um impacto na saúde e social, um ônus econômico muito grande, principalmente com relação à falta no trabalho e perda da produtividade, além da propensão 6,7 vezes maior de utilização dos serviços de saúde ${ }^{12}$ 
Considerada um transtorno multidimensional, 0 tratamento da insônia primária deve ser individualizado, baseado na natureza e gravidade dos sintomas, combinando medidas farmacológicas e não farmacológicas. ${ }^{7,13-14}$

O tratamento farmacológico faz uso principalmente de substâncias psicotrópicas, os ansiolíticos e hipnóticos - sedativos, os quais apresentam potencial para abuso, dependência e efeitos adversos, além de questionamentos quanto à ação eficaz a longo prazo. ${ }^{14-15-16}$

Entre as abordagens não farmacológicas, a terapia cognitiva-comportamental é considerada eficaz na duração e na redução dos sintomas da insônia, mas ainda necessita evidências na melhora das funções diurnas e na qualidade de vida.?

As terapias complementares também são indicadas e utilizadas, mas há poucos estudos relacionando sua prevalência e eficácia. ${ }^{14}$

Diferentes razões levam os insones a utilizar as terapias complementares: para o bem-estar e prevenção de doenças; para tratar condições médicas específicas; por recomendação médica, porque tratamentos convencionais não ajudaram e, devido ao alto custo de tratamentos médicos. ${ }^{17}$

Estas terapias englobam diferentes técnicas que visam à assistência de saúde ao indivíduo, abrangendo a prevenção e o tratamento auxiliar, com uma visão ampliada do processo saúde-doença, através do cuidado global ou holístico, considerando o indivíduo em sua integralidade corpo/mente e reconhecendo sua natureza energética, além da visão de que a saúde ou a doença é gerada por uma pessoa e não causada apenas pelo agente da doença e pela sua evolução. Podem ser classificadas como tecnologias leves, que não se opõem à doença, como nas abordagens biomédicas. ${ }^{18-19-20}$

As terapias complementares mais indicadas na insônia primária são as que incluem formas de relaxamento entre outros procedimentos, por exemplo, as técnicas de massoterapia, yoga e meditação, com o objetivo de diminuir o desamparo afetivo e diminuir o alerta emocional, presentes na insônia primária. ${ }^{14}$
Entre as terapias complementares encontra-se a reflexologia podal, que na China, é conhecida de forma documentada desde o séc. II a.C.. Baseia-se no princípio de que todos os órgãos, glândulas e estruturas ósseas do corpo, estão representados em pontos específicos dos pés. Logo, a pressão adequada nestes pontos, atingirá reflexamente os órgãos correspondentes, provocando mudanças fisiológicas no corpo, movimentando ou acelerando a capacidade natural de reequilíbrio do organismo humano em busca da homeostasia e consequentemente à correção de distúrbios físicos ou emocionais. ${ }^{21-22}$

A reflexologia podal tem mostrado resultados positivos no controle da insônia, mas os estudos ainda são escassos e necessitam melhores delineamentos. ${ }^{23}$

Desta maneira, devido às alterações na saúde física e mental causadas pela insônia, algumas limitações do tratamento convencional e o interesse da população nas terapias complementares, o presente estudo teve o objetivo de verificar os efeitos da reflexologia podal, em voluntárias apresentando alterações do sono.

\section{MATERIAIS E MÉTODOS}

O presente estudo foi aprovado pelo Comitê de Ética em Pesquisa em Seres Humanos, da Universidade Paranaense-Unipar (CAAE-0705.0.375.000-09), em 2012.

As voluntárias foram esclarecidas quanto aos procedimentos de estudo e assinaram o termo de consentimento livre e esclarecido.

Constituiu-se de um estudo de caso clínico, transversal, de natureza quantitativa.

Foram selecionadas quatro voluntárias que preencheram os critérios de inclusão: sexo feminino; idade entre 25 - 45 anos; sedentária; não fumante; sem o hábito de beber; sem fazer uso de medicamentos psicotrópicos; não gestante; saudável, sem varizes ou outros problemas vasculares e sem lesões nos pés.

Para a coleta de dados foi utilizado o Índice de Qualidade do Sono de Pittsburgh - IQSP. Um questionário que se destina a avaliar as características 
dos padrões de sono e quantificar a qualidade do sono, sendo um instrumento com confiabilidade e validade previamente estabelecidas. ${ }^{24-25-26}$

O IQSP é composto por dez questões, algumas com subdivisões, agrupadas em sete componentes de avaliação, cada qual pontuados em uma escala de zero a três, sendo três a pior pontuação.

A avaliação dos componentes do IQSP ocorre da seguinte forma: o primeiro refere-se à qualidade subjetiva do sono, ou seja, a percepção individual a respeito da qualidade do sono; o segundo demonstra a latência do sono, que corresponde ao tempo necessário para iniciar o sono; o terceiro avalia a duração do sono, ou seja, quanto tempo permanece dormindo; o quarto indica a eficiência habitual do sono, obtido por meio da relação entre o número de horas dormidas e o número de horas em permanência no leito, não necessariamente dormindo; o quinto remete aos distúrbios do sono, ou seja, a presença de situações que comprometem as horas de sono; o sexto componente analisa o uso de medicação para dormir; o sétimo é inerente à sonolência diurna e aos distúrbios durante o dia, referindo-se às alterações na disposição e entusiasmo para a execução das atividades rotineiras.

Os escores dos sete componentes são somados para a conferência da pontuação global do IQSP, a qual varia de 1-21, sendo que pontuações de 0-4 indicam boa qualidade do sono; pontuações de 5-10 indicam qualidade ruim, e acima de 10 indicam distúrbios do sono.

Inicialmente foi aplicado o IQSP para a confirmação das alterações do sono relatadas em ficha de avaliação.

A coleta de dados ocorreu no período de agosto a novembro do ano de 2012, com a realização de oito sessões de reflexologia podal, com a duração média de trinta minutos, $1 \mathrm{x} /$ semana, exceto no período menstrual, quando as voluntárias não receberam a técnica, por medida preventiva às possíveis alterações no fluxo menstrual.

Cada sessão foi acompanhada por uma ficha de evolução.

A voluntária B realizou seis sessões pelo motivo de ter iniciado mais tarde no projeto.
As sessões de reflexologia podal foram realizadas por duas acadêmicas participantes do Programa de Iniciação Científica, sendo que cada uma delas permaneceu com duas voluntárias do início ao final do estudo.

A técnica, realizada no laboratório de estética corporal da Unipar, Unidade Paranavaí, abrangeu toda a área dos pés, utilizando o mapa podal completo. ${ }^{27-28}$ Foram padronizadas a sequência, o tempo e a forma de manipulação dos pontos reflexos.

A técnica de aplicação consistiu em estímulos através de pressão com o polegar ou as articulações dos dedos das mãos, em diferentes regiões dos pés, incluindo planta, dorso, borda interna e externa, com as voluntárias deitadas em maca, em decúbito dorsal. As duas terapeutas utilizaram os mesmos procedimentos.

Foi utilizado álcool $70^{\circ}$ G.L. para a higienização dos pés e loção cremosa hidratante contendo óleo de amêndoas e/ou semente de uva, para facilitar o deslizamento.

Ao final do estudo foi aplicado o IQSP para a comparação dos resultados antes e após as sessões de reflexologia podal.

\section{RESULTADOS}

Para a voluntária A, 26 anos, a queixa principal era a dificuldade em iniciar o sono, de acordar várias vezes durante a noite, apresentar sonhos ruins ou pesadelos, fazer uso ocasionalmente de medicação fitoterápica para dormir, além de relatar ser muito ansiosa. Durante o dia apresentava-se sonolenta, cansada e desanimada, sentia-se como se não tivesse dormido. O escore para o IQSP inicialmente foi de quinze, indicando distúrbios do sono.

Realizou oito sessões de reflexologia podal e não relatou desconforto ao toque em nenhuma sessão.

Os benefícios observados, através da ficha de evolução, tiveram início a partir da terceira sessão onde relatou melhora na ansiedade e já conseguir dormir mais rapidamente. A partir da quinta sessão já relatou acordar menos durante a noite e sentir-se mais descansada durante o dia.

Ao final das sessões, não fazia mais uso de medicação fitoterápica para dormir. Sentia-se plena- 
mente satisfeita com os resultados. O IQSP global foi zero, indicando boa qualidade do sono. Esta voluntária apresentou os melhores resultados à técnica.

Para a voluntária B, 40 anos, a queixa principal era de acordar várias vezes no meio da noite, para ir ao banheiro ou pelo próprio ronco, além de apresentar dores musculares principalmente na região de ombros e pernas. Durante o dia sentia dificuldade para manter-se acordada e apresentava-se com falta de ânimo e indisposição. O escore para o IQSP inicialmente foi de onze indicando distúrbios do sono.

Realizou seis sessões.

A voluntária manifestou desconforto ao toque somente na primeira sessão, sentindo dores principalmente no abdômen durante o toque nesta região reflexa dos pés.

Os benefícios observados através da ficha de evolução teve início a partir da quarta sessão onde relatou estar mantendo o sono por mais tempo, acordando menos durante a noite e apresentando menos dores no corpo, apesar de continuar a roncar.

Ao final das sessões o IQSP global foi de quatro (4), indicando qualidade boa do sono.

Para a voluntária $C, 26$ anos, a queixa principal era por demorar em iniciar o sono (quase duas horas), apresentar dores musculares na região cervical e ombros, sentir dificuldade para respirar e ter sensação de frio durante a noite e, além de afirmar ser ansiosa.

Apresentava-se com muita indisposição durante o dia, e pouca sonolência diurna.

O escore para o IQSP inicialmente foi de treze, indicando alterações do sono.

Realizou oito sessões. Os benefícios, observados através da ficha de evolução, teve início a partir da terceira sessão, com diminuição da ansiedade e já iniciar o sono mais rapidamente.

Chegou ao final das sessões com menos dificuldades para respirar, sem a sensação de frio e de dores no corpo.

A voluntária não relatou desconforto ao toque em nenhuma sessão.

Ao final das sessões o IQSP global foi de cinco, indicando qualidade ruim do sono, mas a voluntária declarou sentir-se satisfeita com os resultados posi- tivos na diminuição do tempo de iniciar o sono e por não apresentar mais as dores no corpo.

Logo, no presente estudo, duas voluntárias (A e B), ou seja, $66,6 \%$ da amostra conseguiram ao final das sessões, atingir boa qualidade do sono (escore $\leq 4)$. A voluntária $\mathrm{C}$ passou de transtornos do sono para qualidade ruim do sono (escore 5).

Os dados de uma das voluntárias foram descartados devido à mesma ter tido problemas com os seus dois filhos, necessitando acordar à noite especificamente para atendê-los, o que tornou os dados do estudo equivocados, sendo necessário descartá-los.

As figuras 1,2,3 mostram os escores para cada componente do IQSP e a figura 5 mostra o escore global do IQSP para as três voluntárias.

\section{DISCUSSÃO}

A qualidade do sono representa um fenômeno complexo difícil de definir de medir objetivamente. Inclui aspectos quantitativos do sono: duração, latência, número de despertares e, também abrange aspectos mais subjetivos, isto é, a profundidade do sono e capacidade de reparação do mesmo. Devido à importância e as dificuldades inerentes em definir e quantificar a qualidade do sono, torna-se importante ter um instrumento clínico que afira a qualidade do sono como o IQSP, o qual fornece medida confiável e padronizada da qualidade do sono, consegue discriminar as pessoas que dormem bem e aquelas que dormem mal, ser um instrumento fácil de ser utilizado tanto por leigos, clínicos e pesquisadores e, fornecer um resumo das variedades de distúrbios que podem afetar a qualidade do sono. ${ }^{24}$

Baseado no IQSP, a reflexologia podal, neste estudo de caso mostrou resultados máximos para a voluntária $\mathrm{A}$, atingindo o escore 0 (zero).

Para a voluntária $\mathrm{B}$, os resultados foram positivos finalizando as sessões com boa qualidade do sono, mas, sendo possível ainda, melhorar os escores dos componentes: duração do sono, distúrbios do sono, sonolência e disfunção diurna.

A voluntária $C$ não conseguiu atingir o nível de qualidade boa do sono, mas a queda do escore global do IQSP de 13 para 5, foi muito importante para ela, 
ainda que necessitando melhorar os resultados dos componentes da qualidade subjetiva do sono, latência do sono e da sonolência e disfunção diurna.

Neste sentido, Jeongsoon et $\mathrm{a}^{23}$ realizaram uma análise sistemática e uma meta-análise sobre o efeito da reflexologia sobre o sono, em dezoito trabalhos publicados entre 2000-2009. O estudo indicou que a técnica da reflexologia podal conseguiu atuar como promotora do sono, apesar dos resultados não homogêneos no tamanho dos efeitos.

Ainda, sobre o presente estudo, através da ficha de evolução foram observadas algumas considerações; na primeira sessão a voluntária B relatou sensação desagradável no abdômen quando esta área reflexa no pé foi tocada. De acordo com Fritz $^{29}$, se uma parte do corpo está em desequilíbrio, sua área de projeção no microssistema (pé) também vai revelar esse desequilíbrio, mostrando sinais na pele (dureza, congestão) ou, dor aguda ao toque no ponto correspondente. A partir da segunda sessão, a dor não foi mais observada.

Com relação ao início dos efeitos da reflexologia podal sobre o sono, os mesmos foram observados pelas voluntárias A e C a partir da terceira sessão e para a voluntária $B$, a partir da quarta sessão, de acordo com a ficha de evolução.

Estudos mostram que o início dos benefícios da reflexologia pode variar. Ribeiro ${ }^{30}$ utilizou a técnica no pós-operatório de cirurgia cardíaca em seis pacientes, e conseguiu observar os efeitos positivos através de duas sessões, com duração média de quarenta e cinco minutos, sendo a primeira sessão realizada na UTI e a segunda, na unidade de internação cirúrgica, após a alta da UTI. A técnica contribuiu para que estes pacientes passassem por este momento sentindo-se melhor, proporcionando relaxamento, conforto, sono e repouso, comprovados através de relato dos mesmos.

Já, Gunnarsdottir e Mc-Alpine ${ }^{31}$ observaram que o nível da dor, em quatro das seis mulheres portadoras de fibromialgia, as quais receberam dez sessões de reflexologia podal, diminuiu de intensidade da sétima para a oitava sessão, indicando que foram necessárias várias sessões de reflexologia para se conseguir os resultados positivos significantes e duradouros, já que as mulheres foram acompanhadas para se verificar a continuidade destes benefícios, por mais duas semanas.

No presente estudo foram evidenciados, também, resultados positivos das voluntárias em relação a algumas questões que caracterizam o componente "distúrbios do sono", como a presença de dores (voluntárias B e C), que neste caso, foram relatadas serem devidas às tensões musculares. Para Fritz $^{29}$, quando se realiza a acupressão, sabe-se que muitas terminações nervosas dos pés se correlacionam com estes pontos, os quais quando estimulados favorecem a liberação de endorfinas e encefalinas, mediadores químicos que favorecem o alívio da dor, melhorando a sensação de bem-estar.

Ainda, a reflexologia podal pode ser explicada através do ato reflexo. $\mathrm{O}$ reflexo é o resultado de uma resposta inconsciente e involuntária a um estímulo. ${ }^{32}$

Doughans e Ellis ${ }^{33}$, Ferrer De Dios ${ }^{34}$ afirmam que nos pés passam cerca de 72000 terminações nervosas. No caso da reflexologia podal o estímulo ou sinal proporcionado pela pressão aplicada às áreas reflexas dos pés ativa o impulso nervoso de neurônios sensoriais aferentes que é conduzido até o Sistema Nervoso Central. O cérebro recebe este estímulo, em uma área específica situada na região medial superior onde é codificado e enviado através de nervos eferentes para os órgãos específicos. O resultado é um melhor funcionamento dos órgãos devido à estimulação da circulação sanguínea e linfática, favorecendo a absorção de nutrientes e facilitando a eliminação de toxinas, levando, desta maneira, ao restabelecimento da homeostase.

No caso específico das voluntárias, o problema não era em um órgão específico, mas sim problemas com a qualidade do sono, relacionado a um estresse fisiológico e/ou psicológico, podendo, também ter o benefício da relexologia podal.

Lourenço ${ }^{21}$, Ferrer De Dios ${ }^{34}$ afirmam que, quando a patologia não está relacionada especificamente a um órgão e sim, a desequilíbrios hormonais ou a um agente agressor emocional, a reflexologia podal atuará de forma global no organismo. 
Além disso, Vennells ${ }^{35}$ afirma que além das áreas correspondentes a todo o organismo humano, há uma região, nos pés, que apresenta o ponto do plexo epigástrico ou solar, localizado na parte central dos pés. Este é um emaranhado de ramificações nervosas que se localiza na cavidade abdominal. As áreas reflexas do plexo solar são de grande importância para o relaxamento, contribuindo para uma respiração mais confortável e diminuindo a ansiedade.

A ação da reflexologia podal pode ser explicada, também, através da Medicina Tradicional Chinesa, cujo princípio baseia-se no conceito da Energia vital ou QI, a qual se movimenta no corpo humano, circundando células, tecido muscular e órgãos, através de meridianos ou canais, sendo a fonte integradora e reguladora da forma físico-química, ou seja, a que mantém o organismo vivo e funcionando adequadamente ${ }^{36}$.

Dougans e Ellis ${ }^{33}$ afirmam que de acordo com os conceitos orientais, o desenvolvimento das doenças está relacionado aos desequilíbrios destas forças ou energias que agem no corpo. Para Vennels ${ }^{35}$ quando ocorrer uma estagnação no QI o organismo manifestará este distúrbio como doença ou dor, ou qualquer outro sintoma mental ou físico.

A reflexologia podal tem, então, a particularidade de eliminar os bloqueios ao longo dos meridianos, estimulando o fluxo livre de energia vital e reequilibrando todo o sistema. Ao tocar o ponto de um órgão energeticamente, no pé, estimula-se o canal energético que liga esse ponto ao órgão interno correspondente. ${ }^{33}$

Vennels ${ }^{35}$ afirma, ainda, que a reflexologia podal aumenta a capacidade da pessoa de lidar mais positivamente com as situações estressantes, pela troca de energia positiva por meio do toque e da sensação de bem-estar promovida pela energia revigorada.

Estas teorias que fundamentam a reflexologia podal podem justificar os benefícios da voluntária $\mathrm{A}$ em não apresentar mais sonhos ruins ou pesadelos e a voluntária $\mathrm{C}$, por não apresentar sensação de frio durante o sono e, também, com relação à melhora na disposição e ânimo durante o dia, pelas três voluntárias, ainda que não alcançados os níveis excelentes para as voluntárias $\mathrm{B}$ e C, talvez necessitando maior número de sessões.
Os benefícios da reflexologia podal foram observados neste estudo de caso e em outros trabalhos citados, mas, praticamente não existem trabalhos científicos que comprovem o mecanismo de ação desta técnica. $O$ trabalho de Nakamuru et a ${ }^{37}$ é uma exceção e conseguiu demonstrar a relação entre atividade cortical e a estimulação sensorial de três áreas reflexas do pé esquerdo relacionadas aos olhos, ombro e intestino grosso, utilizando imagem de ressonância magnética. A análise demonstrou que a estimulação reflexológica induziu processo somatosensorial correspondente à área reflexa e que a neuroimagem pode ser utilizada para analisar as bases do mecanismo de ação da reflexologia podal.

Outros estudos como os de Asltoghiri e Ghodsi ${ }^{38}$ conseguiram mostrar que os resultados obtidos com a reflexologia podal são superiores àqueles realizados com massagens não específicas nos pés. Mulheres na menopausa tiveram redução nas alterações do sono $(\mathrm{p}<0,001)$ utilizando como parâmetro $o$ Índice de Qualidade do Sono de Pittsburgh, quando submetidas a vinte e uma sessões de reflexologia podal, diariamente, com duração de quinze minutos, em comparação ao grupo controle que recebeu massagem não específica nos pés.

Apesar do benefício da reflexologia podal na qualidade do sono das voluntárias, torna-se necessário o diagnóstico médico e o tratamento adequado, através de uma equipe multiprofissional.

Os resultados obtidos neste estudo e em outros citados neste trabalho corroboram com os princípios da reflexologia podal de restabelecer o equilíbrio e bem-estar, demonstrando ser uma intervenção efetiva no controle da insônia primária.

Estudos devem ser realizados para um maior entendimento a respeito de seu mecanismo de ação e padronização dos resultados.

\section{CONSIDERAÇÕES FINAIS}

Este estudo de caso demonstrou os benefícios da Reflexologia podal na qualidade do sono de três voluntárias, aferidos pelo escore global do Índice de Qualidade do Sono de Pittsburgh, antes e após as sessões. 
Através do resultado obtido no estudo, observou-se que esta técnica de massagem é promissora na contribuição para o cuidado com a saúde, como recurso complementar importante junto ao tratamento da medicina convencional.

Percebeu-se a importância da discussão na sociedade de terapias que abordam o ser humano de forma holística, para melhor compreensão do processo saúde-doença, de forma a amenizar o preconceito, para o melhor entendimento e para planejamento de formas de inserção destas terapias no sistema de saúde.

Os estudos nesta área devem ser continuados e, realizados através de amostras maiores, com grupo controle, para obtenção de dados estatisticamente significativos, inclusive com acompanhamento da duração dos resultados positivos obtidos.

\section{AGRADECIMENTOS}

Às acadêmicas participantes do Programa de Iniciação Científica-PIC, Carla Maria Rodrigues e Patricia Amara da Silva.
Figuras 1,2,3: Escores dos componentes do IQSP para as voluntárias A,B e C.
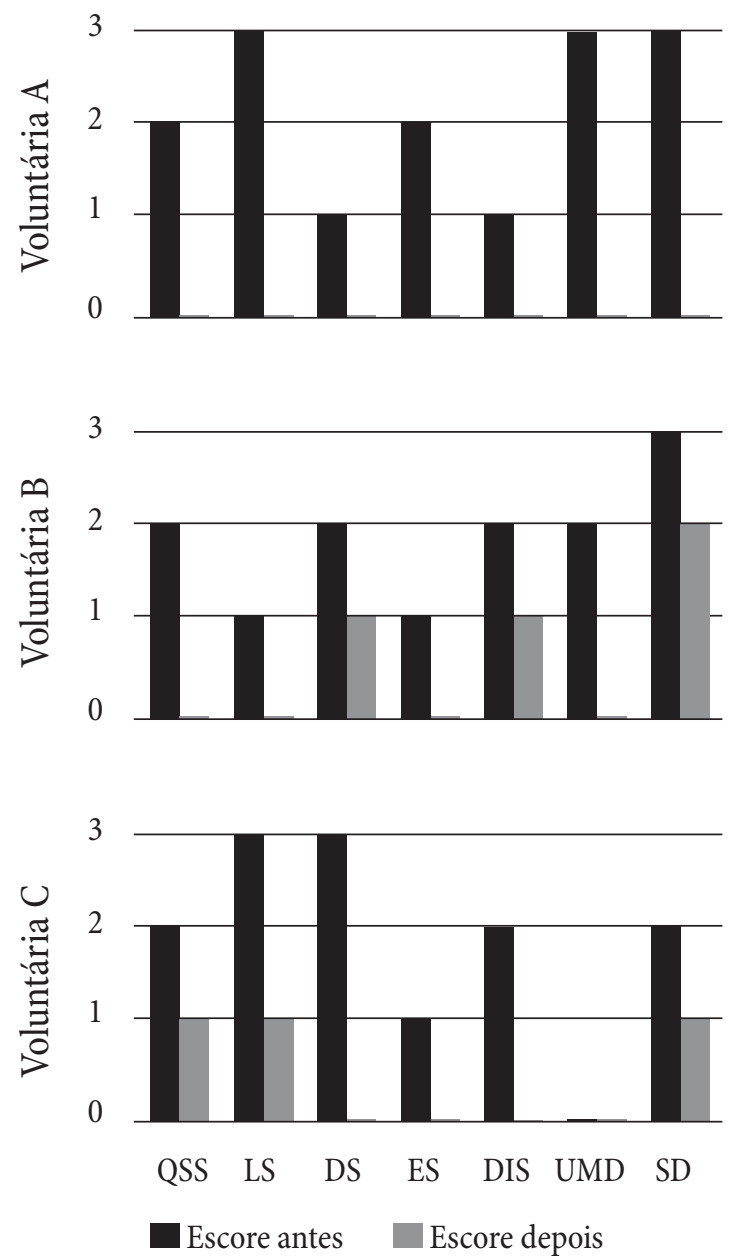

Legenda:

QSS=qualidade subjetiva do sono; LS=latência do sono; DS=distúrbios do sono; ES=eficiência do sono;

$\mathrm{DIS}=$ distúrbios do sono; $\mathrm{UMD}=$ uso de medicação para dormir; $\mathrm{SD}=$ sonolência e disfunção diurna

Figura 4: Comparação dos valores globais do IQSP para as voluntárias A, B, e C, antes e após as sessões de reflexologia podal.

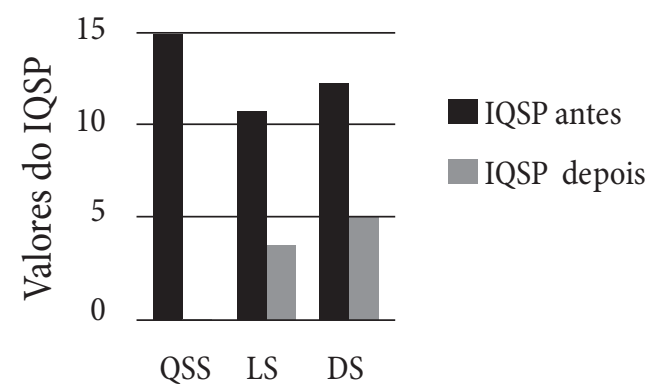




\section{CONFLITO DE INTERESSES}

Declara não haver

\section{FONTE DE FINANCIAMENTO}

Programa de Iniciação Científica, Unipar - Pr.

\section{REFERÊNCIAS}

38 Asltoghiri, M, Ghodsi, Z. The effects of reflexology on sleep disorder in menopausal women. Procedia - Soc Behav Sci. 2012; 31:242-246.

17 Bertisch S, Wells RE, Smith MT, Mc Carthy EP. Use of relaxation techniques and complementary and alternative medicine by american adults with Insomnia symptoms: results from a national survey. J. Clinic. Sleep Med. 2012; 8(6), 681-691.

25 Borges, G.F. Nível de atividade física, capacidade funcional e qualidade de sono de idosas. [Dissertação de mestrado]. Florianópolis: Universidade Federal de Santa Catarina, 2006.

24 Buysse, DJ, Reinolds CF 3rd, Monk TH, Berman SR, Kupfer DJ. The Pittsburg sleep quality Index: a new instrument for psychiatric practice and research. Psychiat. Res. 1989 May; 2(28):193-213.

36 Campiglia H. Psique e medicina tradicional chinesa. São Paulo: Roca; 2004.

11 Drake C, Roehrs T, Roth T. Insomnia causes, consequences, and therapeutics: an overview. Depress Anxiety. 2003; 18(4):163-170

33 Doughans I, Ellis S. Reflexologia: um guia passo a passo para a aplicação da reflexologia. São Paulo: Cultrix; 2001.

34 Ferrer De Dios, R. Reflexology. Rev Enferm. 2005; Mar; 38(3):42-46.

29 Fritz, S. Fundamentos da Massagem Terapêutica. 2. ed. São Paulo: Manole; 2002.

27 Guillandres, A. Reflexologia um guia passo a passo. São Paulo: Manole; 1999.

31 Gunnarsdottir TJ, Mc-Alpine CP. Effects of reflexology on fibromyalgia symtoms: a multiple case study. Complement Therap. Clin. Pract. 2010; 16:167-172.

32 Guyton AC. Tratado de fisiologia médica. 11 ed. Rio de Janeiro: Elsevier; 2006.

23 Jeongsoon NL, Han M, Chung NGY, Kim J, Choi J. Effects of foot reflexology on fadigue, sleep and pain: a systematic review and meta-analysis. J Korean Acad Nurs. 2011 Dec; 41(6):821-833.

21 Lourenço OT. Reflexologia podal. 2.ed. São Paulo: Ground; 2002.

19 Luz MT. Cultura contemporânea e medicina alternativa: novos paradigmas em saúde no fim do século XX. Rev Saúde Coletiva. 2005; 15(5):145-176.

28 Marquardy, H. Reflexoterapia pelos pés. 5ed. São Paulo: Manole, 2005.

2 Martinez D, Lenz MCS, Menna-Barreto L. Diagnosis of circadian rhythm sleep disorders. J Bras Pneumol. 2008 Mar; 34:173-180.

3 McCrae CS, Wilson NM, Lichstein KL, Durrence HH, Taylor DJ, BUSH AJ et al.'Young old' and 'old old' poor sleepers with and without insomnia complaints. J Psychosom Res. 2003 Jan; 1(54):11-1.

8 Monti JM. Insônia primária: diagnóstico diferencial e tratamento. Ver. Bras. Psiquiatr. 2000; 1(22):31-34.

7 Morin CM, Leblanc M, Daley M, Gregoire JP, Mérette C. Epidemiology of insomnia: prevalence, self-help treatments, consulations, and determinants of help-seeking behaviors. Sleep Med. 2006 Mar; 7:123-130.

4 Müller, M.R., Guimarães, S.S. Impacto dos transtornos do sono sobre o funcionamento diário e a qualidade de vida. Estud. Psic. 2007 Out-Dez; 4(24): 519-528.

37 Nakamuru T, Miura N, Fukushima A, Kawashima R. Somatotopical relationship between cortical activity and reflex areas in reflexology: a functional magnetic resonance imaging study. Neurosci Lett. 2008 Dec; 448(1):6-9.
9 Ohayon MM. Epidemiology of insomnia: what we know and what we still need to learn. Sleep Med Rev. 2002 Apr; 6(97):97-111.

5 Palma BD, Tiba PA, Machado RB, Tufik S, Suchecki D. Repercussões imunológicas dos distúrbios do sono: o eixo hipotálamo-ptuitáriaadrenal como fator modulador. Ver. Bras. Psiquiatr. 2007 May; 1(29):s34-s38

15 Passos GS, Tufik S, Santana MG de; Poyares D, Melo MT de. Tratamento não farmacológico para a insônia crônica. Rev. Bras. Psiquiatr. 2007; 29(3):279-282.

14 Pearson NJ, Johnson Ll, Nahhir Rl, Insomnia, trouble sleeping and complementary alternative medicine. Arch Intern Med. 2006 Sept 166(16):1775-1782.

6 Pereira EF, Teixeira CS, Kothe F, Maerino EAD, Daronco LSE. Percepção de qualidade do sono e da qualidade de vida de músicos de orquestras. Rev.Psiq. Clín. 2013.37(2):48-51.

30 Ribeiro JA. O cuidado de enfermagem no pós-operatório de cirurgia cardíaca: as Percepções da pessoa que recebe reflexoterapia. [Dissertação de mestrado]. Florianópolis: Universidade Federal de Santa Catarina; 2009.

13 Ringdahl EM, Pereira SL, Delzell JE Jr. Treatment of primary insomnia. J. Am. Board. Fam. Pract. 2004 may-Jun; 17(3):212-219, 2004

10 Robaina JR, Lopes CS, Rotenberg L, Fischer FM, Moreno CR de. Eventos de vida produtores de estresse e queixas de insônia entre auxiliares de enfermagem de um hospital universitário no Rio de Janeiro: estudos pró-saúde. Rev. Bras. Epidemiol. 2009, 12(3): 501509.

12 Santos-Silva R, Castro LS, Taddei JA, Tufik, S, Bittecourt LRA. Sleep disorders form medical services: evidence from a population-based longitudinal study. PloSONE. $2012 ; 7(2)$ :e30085.

1 Taheri, S.; Zetzer, J. M.; Mignot, E. The role of hypocretins (orexins) in sleep regulation and narcolepsy. Annu. Rev. Neurosci. 2002; 25: 283-313.

22 Tiran, D.; Chummun H. The physiological basis of reflexology and it use as a potential diagnostic tool. Complement. Ther Clin.Pract. 2005 Feb; 11(1):58-64.

20 Tresser CD, Barros NF de. Social medicalization and alternative and complementary medicine: the pluralization of health sources in the Brazilian unified health system. Ver. Saúde Públ. 2008; 42(5):914920.

18 Trovo MM, Silva MEP da. Terapias alternativas/complementares: a visão do graduando de enfermagem. Ver. Esc. Enferm. 2002; 36(1):75-79.

35 Vennells, D. O que é reflexologia? Rio de Janeiro: Nova Era; 2003.

26 Verri FR, Garcia AR, Zuim PRJ, Almeida EO de, Falcon-Antenucci RM, Shibayama R. Avaliação da qualidade do sono em grupos com diferentes níveis de Desordem temporomandibular. Pesq. Bras. Odontoped. Clin. Integr. 2008 Maio-Ago; 2(8):165-169.

16 Xie CL, Gu Y, Wang WW, Lu l, Fu, DL, Liu, AJ. et al. Efficacy and safety of Suanzaoren decoction for primary insomnia: a systematic review of randomized controlled trials. BMC Complement. Altern. Med. 2013; 13-18. 